OPEN ACCESS

Edited by:

Stefania Tacconelli, Università degli Studi G. d'Annunzio Chieti e Pescara, Italy

Reviewed by: Nasiara Karim,

University of Malakand, Pakistan

Carole L. Wilson,

Medical University of South Carolina,

United States

*Correspondence:

Claudia Fabian claudia.fabiani@gmail.com

Luca Cantarin

cantariniluca@hotmail.com

Specialty section: This article was submitted to Inflammation Pharmacology,

a section of the journal

Frontiers in Pharmacology

Received: 24 July 2019 Accepted: 13 November 2019 Published: 09 December 2019

Citation:

Fabiani C, Vitale A, Emmi G, Sgheri A, Lopalco G, Sota J, Guerriero S, lannone F, Frediani B, Vannozzi L, Bianco MT, Giovannini V, Tosi GM and Cantarini L (2019) The Role of Biosimilars in Uveitis: Long-Term Real-World Outcomes of the Switch From Original to Biosimilar TNF-Alpha Inhibitors.

Front. Pharmacol. 10:1468. doi: 10.3389/fphar.2019.01468

\section{The Role of Biosimilars in Uveitis: Long-Term Real-World Outcomes of the Switch From Original to Biosimilar TNF-Alpha Inhibitors}

Claudia Fabiani ${ }^{1 *}$, Antonio Vitale ${ }^{2}$, Giacomo Emmi ${ }^{3}$, Arianna Sgheri ${ }^{1}$, Giuseppe Lopalco ${ }^{4}$, Jurgen Sota ${ }^{2}$, Silvana Guerriero ${ }^{5}$, Florenzo lannone ${ }^{4}$, Bruno Frediani ${ }^{2}$, Lorenzo Vannozzi ${ }^{6}$, Maria Teresa Bianco ${ }^{7}$, Valtere Giovannini ${ }^{8}$, Gian Marco Tosi ${ }^{2}$ and Luca Cantarini ${ }^{3 *}$

${ }^{1}$ Ophthalmology Unit, Department of Medicine, Surgery and Neuroscience, University of Siena, Siena, Italy, ${ }^{2}$ Rheumatology Unit, Department of Medical Sciences, Surgery and Neurosciences, University of Siena, Siena, Italy, ${ }^{3}$ Department of Experimental and Clinical Medicine, University of Florence, Florence, Italy, ${ }^{4}$ Rheumatology Unit, Department of Emergency and Organ Transplantation (DETO), University of Bari, Bari, Italy, ${ }^{5}$ Department of Ophthalmology and Otolaryngology, University of Bari, Bari, Italy, ${ }^{6}$ Department of Surgery and Translational Medicine, Eye Clinic, University of Florence, Florence, Italy, ${ }^{7}$ Pharmacy Unit, Siena University Hospital "Santa Maria alle Scotte", Siena, Italy, ${ }^{8}$ Azienda Ospedaliera Universitaria Senese, Le Scotte Hospital, Siena, Italy

Background: Recent expiry of patents for tumor necrosis factor (TNF)- $\alpha$ inhibitors has led to the employment of biosimilars in clinical practice. The aim of the study was to identify any change in the control of ocular inflammatory manifestations among patients with noninfectious uveitis switching from an originator to a corresponding anti-TNF- $\alpha$ biosimilar.

Methods: Thirty-seven consecutive patients (62 eyes involved) with non-infectious uveitis undergoing the switch from anti-TNF- $\alpha$ originators to biosimilars were retrospectively enrolled; the frequency of ocular flares before and after the switch as well as best corrected visual acuity (BCVA), central macular thickness (CMT), daily systemic corticosteroid intake, and frequency of uveitic macular edema (UME) at the switch and at the following assessments were statistically analysed.

Results: The number of ocular flares during the 12 months preceding the switch was 16, corresponding to 3.6 flares/100 patients/12 months; the number of flares after the switch was 14 , corresponding to 2.0 flares/100 patients/12 months. No statistically significant differences were identified in the frequency of flares $(p=0.84)$ and in the number of patients experiencing ocular flares $(p=0.39)$ between the twelve months preceding the switch and the period thereafter. No statistically significant changes were observed in the BCVA $(p=0.27)$, CMT $(p=0.50)$, frequency of UME $(p=0.57)$ and daily corticosteroid intake $(p=0.42)$ between the time of the switch and the last follow-up visit.

Conclusions: The switch to biosimilars represents a feasible treatment choice associated with the maintenance of clinical efficacy in patients with non-infectious uveitis previously treated with the corresponding originator anti-TNF- $\alpha$ biologic agents.

Keywords: biosimilar, benepali, flixabi, imraldi, inflectra, originator, switch 


\section{INTRODUCTION}

Anti-tumor necrosis factor (TNF)- $\alpha$ biologic agents have shown to represent a primary option for the treatment of patients with non-infectious uveitis, especially in refractory cases (Fabiani et al., 2017; Bitossi et al., 2019; Fabiani et al., 2019a; Fabiani et al., 2019b; Fabiani et al., 2019c; Tosi et al., 2019). In particular, the monoclonal anti-TNF- $\alpha$ antibodies adalimumab (ADA) and infliximab (IFX) have been widely used to control intraocular inflammation, prevent ocular structural complications, avoid the impairment of visual function and allow a rapid tapering of systemic corticosteroids (Levy-Clarke et al., 2014; Dick et al., 2018).

Recent expiry of patents for anti-TNF- $\alpha$ originator biologic agents has led to development of similar versions of the original biopharmaceutical products, termed biosimilars. In particular, CT-P13 has been the first biosimilar monoclonal antibody to enter the market with the trade names of Inflectra ${ }^{\circledR}$ or Remsima ${ }^{\circledR}$. Later, additional biosimilar agents have been registered, including Flixabi $^{\circledR}$ (SB2, another biosimilar infliximab), Benepali ${ }^{\circledR}$ (SB4, biosimilar etanercept) and Imraldi $^{\circledR}$ (SB5, biosimilar adalimumab). Physicochemical, pre-clinical, and clinical studies have proved similar efficacy, safety and immunogenicity between originators and biosimilars, thus leading to the approval of biosimilars for all indications of the reference product (Park et al., 2013; Yoo et al., 2013; Cho et al., 2016; Hong et al., 2017; Lee et al., 2019).

Beyond the significant costs associated with the use of originators, the development of biosimilar products has recently accounted for considerable cost savings and a substantial favorable impact on the healthcare budget; from this perspective, the number of patients possibly benefiting from an early access to biological treatment could be increased (Aladul et al.,2019). Nevertheless, introducing biosimilar agents to naïve patients and switching from the originator to a biosimilar gives rise to different medical liability issues. Indeed, switching from an option where the individual response is known to an option where it is unknown may expose patients to unpredictable risks, including loss of efficacy (Rocco et al., 2018). To date, studies addressing the consequences of the switch from originators to biosimilars have included patients with rheumatoid or psoriatic arthritis, spondyloarthritis and inflammatory bowel diseases (Emery et al., 2017; Jørgensen et al., 2017; Smolen et al., 2018; Weinblatt et al., 2018). Conversely, data related to patients with non-infectious uveitis are limited and the issue about ocular disease control after the switch is currently unexplored. In this context, the present study aimed at shedding light on this matter.

\section{MATERIAL AND METHODS}

Patients suffering from non-infectious uveitis consecutively undergoing the switch from anti-TNF- $\alpha$ originator to biosimilar biologic agents were retrospectively enrolled; their demographic, clinical and therapeutic data were collected by accessing patients' medical records.
The aim of the study was to identify any change in the control of ocular inflammatory manifestations in patients with noninfectious uveitis after the switch from an originator anti-TNF- $\alpha$ biologic agent to a corresponding biosimilar. The endpoints of the study corresponded to the identification of any: i) statistically significant difference in the frequency of ocular flares during the follow-up after the switch compared to the preceding 12 months by standardizing the rate of uveitis relapses as number of flares/ 100 patients/12 months; ii) statistically significant changes in the best corrected visual acuity (BCVA) and systemic corticosteroid intake ( $\mathrm{mg} /$ day of prednisone or equivalent) between the time of the switch and the last follow-up visit; iii) statistically significant changes in the mean central macular thickness (CMT) and in the frequency of eyes with uveitic macular edema (UME) or retinal vasculitis at the last assessment (3-, 6- and 12-month follow-up visits) compared with the time of the switch.

The BCVA was expressed in decimal fractions by Snellen charts; CMT was assessed at optical coherence tomography performed at each follow-up visit; UME was diagnosed when CMT was $>300 \mu \mathrm{m}$ and presence of intraretinal fluid was confirmed after regular evaluation of the macular area. Active retinal vasculitis was diagnosed in case of evidence of retinal vascular leakage at fluorescein angiography. The anatomical and clinical classification of uveitis was established according with the standardization of uveitis nomenclature criteria (Jabs et al., 2005). Ocular flare was meant as an acute ocular inflammatory exacerbation occurred after a period of remission.

Descriptive statistics included sample size, percentages, means, and standard deviations. Normality distribution of quantitative data was assessed by using Shapiro-Wilk test followed by pair wise comparisons with unpaired two-tailed $t$ test or Mann-Whitney two tailed U test, as appropriate. For categorical variables, comparisons were performed with Fisher exact test for $2 \times 2$ or $2 \times 3$ contingency tables. Two tailed $p$ values $<0.05$ were considered statistically significant.

The study has been approved by the local Ethics Committee of Azienda Ospedaliera Universitaria Senese, Siena, Italy (Ref. N. 14951). The study protocol was conformed to the tenets of the Declaration of Helsinki; informed consent was obtained from all patients enrolled.

\section{RESULTS}

Thirty-seven patients ( 21 males; 16 females) with a history of uveitis (62 eyes involved) were switched from originator antiTNF- $\alpha$ biological agents to biosimilars as follows: Imraldi $^{\circledR}(\mathrm{n}=$ 20 patients, 33 eyes involved); Flixabi ${ }^{\circledR}$ ( $n=10$ patients, 16 eyes involved); Inflectra ${ }^{\circledR}\left(\mathrm{n}=5\right.$ patients, 9 eyes involved); Benepali ${ }^{\circledR}$ ( $\mathrm{n}=2$ patients, 4 eyes involved). The follow-up duration of patients after the switch to biosimilars was 3 months in 20 patients, 6 months in 6 patients, 12 months in 11 patients. Table 1 summarizes further demographic, clinical and therapeutic features of patients enrolled.

Ocular flares involved 9 patients ( 5 treated with Remicade ${ }^{\circledR}, 3$ with Humira ${ }^{\circledR}$ and 1 with Enbrel ${ }^{\circledR}$ ) during the 12 months 
TABLE 1 | Demographic, clinical and therapeutic features of patients enrolled.

\begin{tabular}{|c|c|}
\hline Patients' features & Years, mean \pm SD \\
\hline Age at the switch & $43.8 \pm 11.9$ \\
\hline Age at uveitis onset & $31.9 \pm 12.7$ \\
\hline Ocular disease duration & $11.4 \pm 7.1$ \\
\hline Age at systemic disease onset & $13.8 \pm 12.4$ \\
\hline Systemic disease duration & $11.7 \pm 8.7$ \\
\hline Anatomical ocular involvement & $\begin{array}{l}\text { Number of eyes } \\
\text { involved, (\%) }\end{array}$ \\
\hline Anterior Uveitis & $18(29)$ \\
\hline Intermediate Uveitis & $2(3.2)$ \\
\hline Posterior Uveitis & $23(37.1)$ \\
\hline Panuveitis & 19 (30.6) \\
\hline History of retinal vasculitis & $16(25.8)$ \\
\hline Systemic disease's features & Number of patients, (\%) \\
\hline Systemic disease related to uveitis & $32(86.5)$ \\
\hline Behçet's disease & $26(70.3)$ \\
\hline Spondiloarthritis & $5(13.5)$ \\
\hline Juvenile idiopathic arthritis & $1(2.7)$ \\
\hline Idiopathic uveitis & $5(13.5)$ \\
\hline \multicolumn{2}{|c|}{ Information about medications at the time of the switch } \\
\hline $\begin{array}{l}\text { Duration of treatment with originators, months } \\
\text { (mean } \pm \mathrm{SD} \text { ) }\end{array}$ & $52.5 \pm 38.9$ \\
\hline Concomitant cDMARDs, n (\%) & $10(27)$ \\
\hline Methotrexate, n (\%) & $4(10.8)$ \\
\hline Cyclosporine A, n (\%) & $4(10.8)$ \\
\hline Azathioprine, n (\%) & $2(5.4)$ \\
\hline $\begin{array}{l}\text { Corticosteroids (prednisone or equivalent), } \\
\text { mg/day (mean } \pm \text { SD) }\end{array}$ & $4.1 \pm 1.9$ \\
\hline
\end{tabular}

$\overline{c D M A R D s, \text { conventional disease modifying anti-rheumatic drugs; } S D \text {, standard deviation. }}$

preceding the switch to biosimilars and 6 (all treated with Flixabi $^{\circledR}$ ) patients thereafter. The number of ocular flares during the 12 months preceding the switch was 16 , corresponding to 3.6 flares/100 patients/12 months; the number of flares after the switch to biosimilars was 14 , corresponding to 2.0 flares/100 patients/12 months. No statistically significant differences were identified in the frequency of ocular flares $(p=0.84)$ and in the number of patients experiencing ocular flares $(p=0.39)$ between the twelve months preceding the switch and the period thereafter.

Ocular flares occurred while on Flixabi ${ }^{\circledR}$ administration in all cases. None of the ocular flares developed within 3-month assessment; 12/14 (85.7\%) ocular flares developed between 3month and 6-month assessments; $2 / 14$ (14.3\%) ocular flares were observed between 6 -month and 12-month follow-up visits. Table 2 describes clinical features of eyes with uveitis at the time of the switch distinguished according with the biosimilar employed.

Figure 1 illustrates percentage of subjects experiencing ocular flares after the switch among patients with and without flares during the previous 12 months. No statistically significant differences were observed between the two groups $(p=1.000)$. Similarly, no statistically significant differences were observed in the frequency of flares preceding the switch between patients with and without flares afterward (17\% and $26 \%$, respectively, $p=1.000)$.

The mean BCVA was $8.4 \pm 2.5$ decimals at the time of the switch and $8.5 \pm 2.48$ decimals at the last assessment $(p=0.27)$.

Retinal vasculitis was found in 3 patients ( 6 eyes, all treated with infliximab) at baseline; $4 / 6$ eyes had an associated UME. All

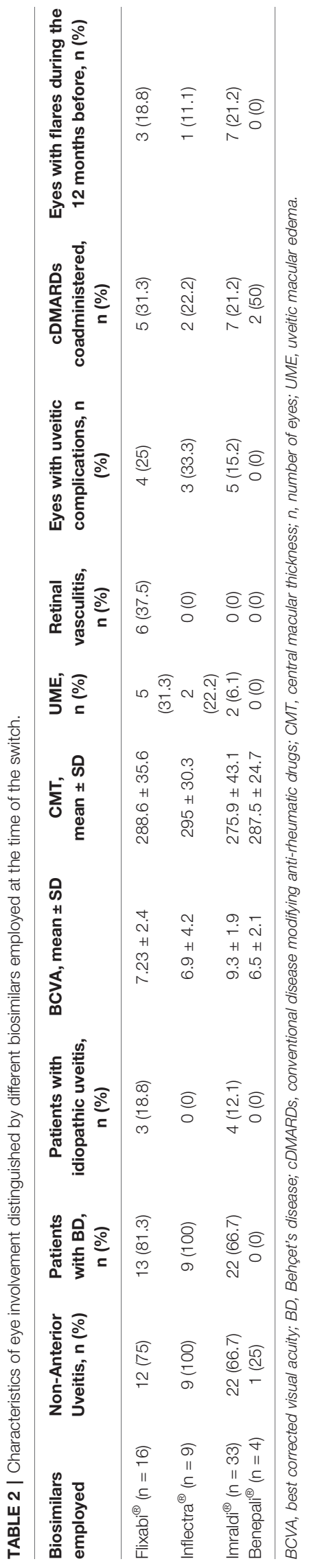




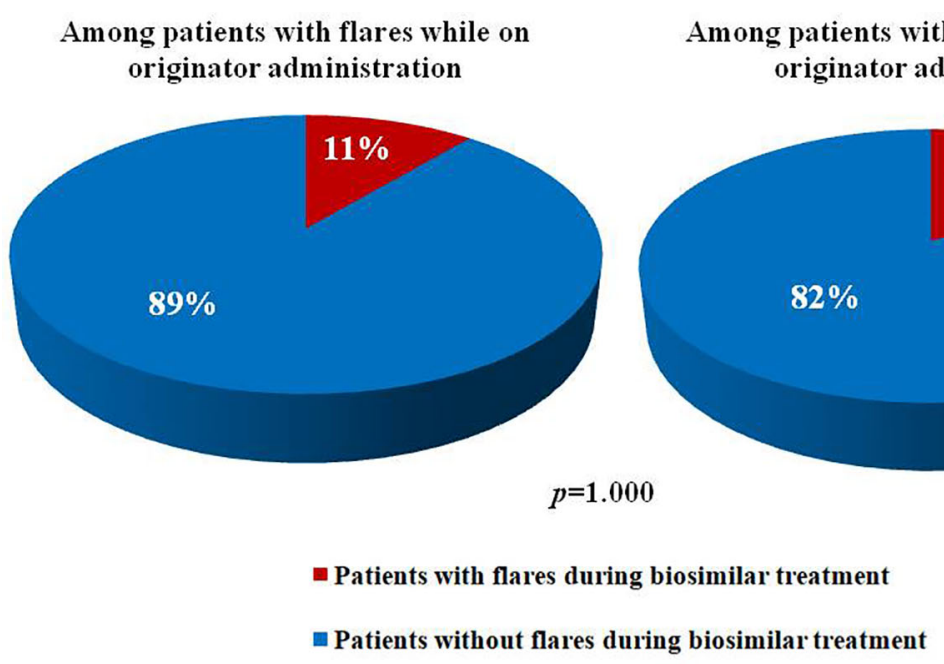

FIGURE 1 | Pie charts illustrates percentages of patients experiencing and not experiencing ocular flares after the switch from an originator anti-tumor necros factor$\alpha$ to a corresponding biosimilar among patients with and without uveitic flares during the 12 months before.

of these patients received $1 \mathrm{mg} / \mathrm{kg} /$ day of oral prednisone at the time of the switch. In all cases oral prednisone was gradually tapered to $\leq 5 \mathrm{mg} /$ day within the first 3 months of follow-up. At the end of the study all of these patients had a follow-up equal or superior to 12 months with no relapses of retinal vasculitis at 3-, 6-, 12-month and last follow-up assessments.

The mean CMT was $281.4 \pm 39.4 \mu \mathrm{m}$ at baseline, $282.9 \pm 31.6$ $\mu \mathrm{m}$ at 3 -month assessment, $275.5 \pm 24.3 \mu \mathrm{m}$ at 6 -month evaluation, $275.9 \pm 27.5 \mu \mathrm{m}$ at 12 -month follow-up visit $(p=$ 0.50 ); UME was observed in 5 patients (9 eyes) at baseline and in one patient (2 eyes) at 3-, 6-, and 12-month assessments ( $p=0.57$ ).

Table 3 provides information about BCVA and CMT as well as the frequency of UME and ocular relapses according to the different follow-up duration of patients enrolled.

The mean daily corticosteroid dosage was $4.1 \pm 1.9 \mathrm{mg} /$ day at the time of the switch and $6.3 \pm 7.8 \mathrm{mg} /$ day at the last follow-up visit $(p=0.42)$. Nine patients took corticosteroids at the start of biosimilar agents and 8 patients at the last assessment $(p=0.78)$. At the time of the switch 10 patients (27.03\%) were concomitantly treated with cDMARDs; at the last assessment, patients administered with cDMARDs were 9 (24.3\%) $(p=0.79)$. Regarding the posology changes, an increase in the frequency of administration was required in 1 patient undergoing Inflectra ${ }^{\circledR}$ treatment; conversely, 3 patients $\left(2\right.$ treated with Flixabi ${ }^{\circledR}$ and 1 treated with Inflectra $\left.{ }^{\circledR}\right)$ decreased the frequency of administration and 1 patient treated with Flixabi ${ }^{\circledR}$ underwent a decrease of the dosage from $5 \mathrm{mg} / \mathrm{kg} / 8$ weeks to $3 \mathrm{mg} / \mathrm{kg} / 8$ weeks. Conversely, no posology changes had been performed during the 6 months preceding the switch.

At the time of the switch, ocular structural complications as consequence of uveal inflammation were present in 9 patients (epiretinal membranes in 7 eyes; macular hole in 2 eyes; retinal atrophy in 2 eyes; retinal atrophy and detachment of retinal pigment epithelium in 1 eye). In addition, age-related macular degeneration had been observed in 1 eye. At the last assessment new uveitic ocular complications arose in 2 patients (3 eyes) treated with Imraldi ${ }^{\circledR}$. In particular, epiretinal membranes were observed in 2 eyes and lens opacity in 1 eye.

No severe adverse events were observed following the switch to biosimilar agents.

\section{DISCUSSION}

The issue about clinical consequences of switching from originators to biosimilar biologic agents currently represents a central topic for scientific debate. However, this topic has mainly been concentrated on patients with rheumatoid or psoriatic arthritis, spondyloarthritis, and inflammatory bowel diseases. On the contrary, data about the switch in patients with noninfectious uveitis are quite scarce in spite of the increasing importance of the anti-TNF- $\alpha$ agents adalimumab and infliximab for the treatment of non-infectious uveitis (Fabiani et al., 2018).

Our results suggest an optimal maintenance of uveitis control following the switch from originators to biosimilars. In particular, the rate of uveitic flares observed during the 12 months preceding the switch and the subsequent follow-up period were comparable (3.6 and 2.0 flares/100 patients/12 months, respectively) with no statistically significant differences. Similarly, visual acuity was maintained at the last follow-up visit compared to the time of the switch. Furthermore, the CMT values and the frequency of UME did not change significantly after the switch. Likewise, the mean daily corticosteroid intake did not change significantly, thus ensuring the persistence of the corticosteroid sparing effect during treatment with anti-TNF- $\alpha$ biosimilars.

Retinal vasculitis was identified bilaterally in 3 patients successfully treated with short-term oral corticosteroids at the time of the switch. Of note, no relapses of retinal vasculitis were 
identified thereafter (the follow-up while on biosimilar treatment lasted at least 12 months in all three cases).

As a whole, our results confirm previous findings obtained from a prospective observational study conducted in 8 patients with uveitis followed for a mean period of 2.9 years (Avouac et al., 2018). In particular, according to Avouac et al. (2018), no statistically significant changes were observed in the uveitis activity score and in the number of ocular flares between the time of the switch and the last visit. In the same way, basing on data from 13 patients with Behçet's disease (10 of them with uveitis), Lopalco et al. (2018) found that switching from reference infliximab to biosimilar CTP-13 was a feasible and uneventful possibility, with no ocular relapses and no significant changes of disease activity during a nine-month follow-up period.

Of note, in our study the frequency of patients with ocular flares after the switch was similar among patients with and without flares during the 12 months preceding the switch. In the same way, the frequency of ocular flares prior to the switch was comparable between patients with and without flares thereafter. As a consequence, the probability of experiencing ocular flares after the switch appears independent of disease control prior to the switch. These findings do not contradict the possibility of ocular flares in patients experiencing full therapeutic success with originators, as previously reported by Cantini et al. (2017).

Noteworthy, in the present study all of the ocular flares recorded after the switch were observed while on the Flixabi ${ }^{\circledR}$ administration. Further studies specifically addressing this issue will have to clarify whether this finding is a result of chance. However, the worst performance obtained with Flixabi ${ }^{\circledR}$ could be related to the worst-case ocular conditions recorded at the time of the switch among patients undergoing this biosimilar infliximab. Indeed, as observed in Table 2, the frequency of retinal vasculitis, UME, Behçet's disease diagnosis and nonanterior uveitis supports a more severe baseline clinical context among patients switched to Flixabi ${ }^{\circledR}$ (Tugal-Tutkun, 2009; Dick et al., 2016; Fardeau et al., 2016; Dick et al., 2018).

The main limitation of the study is represented by its retrospective design. In addition, our study did not include an evaluation of multiple therapy switches between the biosimilar and the originator product or between different biosimilars. Nevertheless, to the best of our knowledge this is the first study primarily addressing the impact of switching from originators to biosimilars in terms of frequency of ocular flares, visual acuity, CMT, daily corticosteroid dosage and occurrence of UME in a fair number of patients with non-infectious uveitis.

In conclusion, the switch to biosimilars represents a feasible treatment choice associated with the maintenance of clinical efficacy in patients with non-infectious uveitis previously treated with the corresponding originator anti-TNF- $\alpha$ biologic agents.

\section{DATA AVAILABILITY STATEMENT}

The datasets generated for this study are available on request to the corresponding author. 


\section{ETHICS STATEMENT}

The studies involving human participants were reviewed and approved by Azienda Ospedaliera Universitaria Senese. The patients/participants provided their written informed consent to participate in this study.

\section{REFERENCES}

Aladul, M. I., Fitzpatrick, R. W., and Chapman, S. R. (2019). The effect of new biosimilars in rheumatology and gastroenterology specialities on UK healthcare budgets: results of a budget impact analysis. Res. Social. Adm. Pharm. 15, 310-317. doi: 10.1016/j.sapharm.2018.05.009

Avouac, J., Moltó, A., Abitbol, V., Etcheto, A., Salcion, A., Gutermann, L., et al. (2018). Systematic switch from innovator infliximab to biosimilar infliximab in inflammatory chronic diseases in daily clinical practice: the experience of Cochin University Hospital, Paris, France. Semin. Arthritis. Rheumatol. 47, 741-748. doi: 10.1016/j.semarthrit.2017.10.002

Bitossi, A., Bettiol, A., Silvestri, E., Di Scala, G., Bacherini, D., Lopalco, G., et al. (2019). Adalimumab accounts for long-term control of noninfectious uveitis also in the absence of concomitant DMARD treatment: a multicenter retrospective study. Mediators Inflamm. 10, 1623847. doi: 10.1155/2019/1623847

Cantini, F., Niccoli, L., Nannini, C., Cassarà, E., and Kaloudi, O. (2017). Rapid loss of efficacy of biosimilar infliximab in three patients with Behçet's disease after switching from infliximab originator. Eur. J. Rheumatol. 4, 288-290. doi: 10.5152/eurjrheum.2017.16112

Cho, I. H., Lee, N., Song, D., Jung, S. Y., Bou-Assaf, G., Sosic, Z., et al. (2016). Evaluation of the structural, physicochemical, and biological characteristics of SB4, a biosimilar of etanercept. MAbs. 8, 1136-1155. doi: 10.1080/19420862.2016.1193659

Dick, A. D., Tundia, N., Sorg, R., Zhao, C., Chao, J., Joshi, A., et al. (2016). Risk of ocular complications in patients with noninfectious intermediate uveitis, posterior uveitis, or panuveitis. Ophthalmology. 123, 655-662. doi: 10.1016/j.ophtha.2015.10.028

Dick, A. D., Rosenbaum, J. T., Al-Dhibi, H. A., Belfort, R. Jr, Brézin, A. P., Chee, S. P., et al. (2018). Guidance on noncorticosteroid systemic immunomodulatory therapy in noninfectious uveitis: fundamentals of care for uveitis (FOCUS) initiative. Ophthalmology. 125, 757-773. doi: 10.1016/j.ophtha.2017.11.017

Emery, P., Vencovský, J., Sylwestrzak, A., Leszczyński, P., Porawska, W., Stasiuk, B., et al. (2017). Long-term efficacy and safety in patients with rheumatoid arthritis continuing on SB4 or switching from reference etanercept to SB4. Ann. Rheum Dis. doi: 10.1136/annrheumdis-2017-211591

Fabiani, C., Vitale, A., Emmi, G., Vannozzi, L., Lopalco, G., Guerriero, S., et al. (2017). Efficacy and safety of adalimumab in Behçet's disease-related uveitis: a multicenter retrospective observational study. Clin. Rheumatol. 36, 183-189. doi: 10.1007/s10067-016-3480-x

Fabiani, C., Sota, J., Vitale, A., Rigante, D., Emmi, G., Vannozzi, L., et al. (2018). Cumulative retention rate of adalimumab in patients with Behçet's diseaserelated uveitis: a four-year follow-up study. Br. J. Ophthalmol. 102, 637-641. doi: 10.1136/bjophthalmol-2017-310733

Fabiani, C., Vitale, A., Rigante, D., Emmi, G., Lopalco, G., Sota, J., et al. (2019a). Efficacy of anti-tumour necrosis factor- $\alpha$ monoclonal antibodies in patients with non-infectious anterior uveitis. Clin. Exp. Rheumatol. 37, 301-305.

Fabiani, C., Vitale, A., Emmi, G., Bitossi, A., Lopalco, G., Sota, J., et al. (2019b). Long-term retention rates of adalimumab and infliximab in non-infectious intermediate, posterior, and panuveitis. Clin. Rheumatol. 38, 63-70. doi: 10.1007/s10067-018-4069-3

Fabiani, C., Sota, J., Vitale, A., Emmi, G., Vannozzi, L., Bacherini, D., et al. (2019c). Ten-year retention rate of infliximab in patients with behçet's disease-related uveitis. Ocul. Immunol. Inflamm. 27, 34-39. doi: 10.1080/09273948.2017.1391297

Fardeau, C., Champion, E., Massamba, N., and LeHoang, P. (2016). Uveitic macular edema. Eye (Lond). 30, 1277-1292. doi: 10.1038/eye.2016.115

Hong, J., Lee, Y., Lee, C., Eo, S., Kim, S., Lee, N., et al. (2017). Physicochemical and biological characterization of SB2, a biosimilar of Remicade ${ }^{\circledR}$ (infliximab). MAbs. 9, 364-382. doi: 10.1080/19420862.2016.1264550

Jabs, D. A., Nussenblatt, R. B., and Rosenbaum, J. T. (2005). Standardization of uveitis nomenclature for reporting clinical data. Results of the First International Workshop. Am. J. Ophthalmol. 140, 509-516. doi: 10.1016/j.ajo.2005.03.057

\section{AUTHOR CONTRIBUTIONS}

$\mathrm{CF}$ and $\mathrm{AV}$ wrote the manuscript. LCand CF designed the study. CF, LC, MTB, and VG finally revised the manuscript. AV performed the data analysis. CF, LC, AV, GMT, JS, SG, GL, AS, GE, FI, BF, and LV took care of patients' enrollment, follow-up of the patients, and data collection.

Jørgensen, K. K., Olsen, I. C., Goll, G. L., Lorentzen, M., Bolstad, N., Haavardsholm, E. A., et al. (2017). Switching from originator infliximab to biosimilar CT-P13 compared with maintained treatment with originator infliximab (NOR-SWITCH): a 52-week, randomised, double-blind, noninferiority trial. Lancet. 389, 2304-2316. doi: 10.1016/S0140-6736(17) 30068-5

Lee, N., Lee, J. J., Yang, H., Baek, S., Kim, S., Kim, S., et al. (2019). Evaluation of similar quality attribute characteristics in SB5 and reference product of adalimumab. MAbs. 11, 129-144. doi: 10.1080/19420862.2018.1530920

Levy-Clarke, G., Jabs, D. A., Read, R. W., Rosenbaum, J. T., Vitale, A., and Van Gelder, R. N. (2014). Expert panel recommendations for the use of antitumor necrosis factor biologic agents in patients with ocular inflammatory disorders. Ophthalmology. 121, 785-96.e3. doi: 10.1016/j.ophtha.2013.09.048

Lopalco, G., Venerito, V., Cantarini, L., Emmi, G., Prisco, D., and Iannone, F. (2018). Long-term effectiveness and safety of switching from originator to biosimilar infliximab in patients with Behçet's disease. Intern. Emerg. Med. 14, 719-722. doi: 10.1007/s11739-018-1970-3

Park, W., Hrycaj, P., Jeka, S., Kovalenko, V., Lysenko, G., Miranda, P., et al. (2013). A randomised, double-blind, multicentre, parallel-group, prospective study comparing the pharmacokinetics, safety, and efficacy of CT-P13 and innovator infliximab in patients with ankylosing spondylitis: the PLANETAS study. Ann. Rheumatol. Dis. 72, 1605-1612. doi: 10.1136/annrheumdis-2012-203091

Rocco, P., Selletti, S., and Minghetti, P. (2018). Biosimilar switching and related medical liability. J. Forensic. Leg. Med. 55, 93-94. doi: 10.1016/j.jflm.2018.02.018

Smolen, J. S., Choe, J. Y., Prodanovic, N., Niebrzydowski, J., Staykov, I., Dokoupilova, E., et al. (2018). Safety, immunogenicity and efficacy after switching from reference infliximab to biosimilar SB2 compared with continuing reference infliximab and SB2 in patients with rheumatoid arthritis: results of a randomised, double-blind, phase III transition study. Ann. Rheumatol. Dis. 77, 234-240. doi: 10.1136/annrheumdis-2017-211741

Tosi, G. M., Sota, J., Vitale, A., Rigante, D., Emmi, G., Lopalco, G., et al. (2019). Efficacy and safety of certolizumab pegol and golimumab in the treatment of non-infectious uveitis. Clin. Exp. Rheumatol. 37, 680-683.

Tugal-Tutkun, I. (2009). Behçet's Uveitis. Middle. East. Afr. J. Ophthalmol. 16, 219-224. doi: 10.4103/0974-9233.58425

Weinblatt, M. E., Baranauskaite, A., Dokoupilova, E., Zielinska, A., Jaworski, J. Racewicz., A., et al. (2018). Switching from reference adalimumab to SB5 (Adalimumab Biosimilar) in patients with rheumatoid arthritis: fifty-two-week phase III randomized study results. Arthritis. Rheumatol. 70, 832-840. doi: 10.1002/art.40444

Yoo, D. H., Hrycaj, P., Miranda, P., Ramiterre, E., Piotrowski, M., Shevchuk, S., et al. (2013). A randomised, double-blind, parallel-group study to demonstrate equivalence in efficacy and safety of CT-P13 compared with innovator infliximab when coadministered with methotrexate in patients with active rheumatoid arthritis: the PLANETRA study. Ann. Rheumatol. Dis. 72, 16131620. doi: 10.1136/annrheumdis-2012-203090

Conflict of Interest: The authors declare that the research was conducted in the absence of any commercial or financial relationships that could be construed as a potential conflict of interest.

Copyright (c) 2019 Fabiani, Vitale, Emmi, Sgheri, Lopalco, Sota, Guerriero, Iannone, Frediani, Vannozzi, Bianco, Giovannini, Tosi and Cantarini. This is an open-access article distributed under the terms of the Creative Commons Attribution License (CC BY). The use, distribution or reproduction in other forums is permitted, provided the original author(s) and the copyright owner(s) are credited and that the original publication in this journal is cited, in accordance with accepted academic practice. No use, distribution or reproduction is permitted which does not comply with these terms. 